Mens

Revue d'histoire intellectuelle et culturelle

mens

\title{
Comment se débarrasser de la Grande Noirceur sans se débarrasser du passé québécois ?
}

\section{Jean-François Laniel et Joseph Yvon Thériault}

Volume 18, numéro 2, printemps 2018

La Grande Noirceur

URI : https://id.erudit.org/iderudit/1066261ar

DOI : https://doi.org/10.7202/1066261ar

Aller au sommaire du numéro

Éditeur(s)

Centre de recherche en civilisation canadienne-française

ISSN

1492-8647 (imprimé)

1927-9299 (numérique)

Découvrir la revue

Citer cet article

Laniel, J.-F. \& Thériault, J. Y. (2018). Comment se débarrasser de la Grande Noirceur sans se débarrasser du passé québécois ? Mens, 18(2), 67-107.

https://doi.org/10.7202/1066261ar
Résumé de l'article

La Grande Noirceur est fille de la Révolution tranquille. Pour affirmer la victoire de la modernité, les nouvelles élites québécoises noircirent exagérément le moment qu'elles venaient de quitter. Les historiens dits révisionnistes sonnèrent l'alarme : le passé québécois aurait été de tout temps " normal ». C'est à cette lecture qu'une nouvelle génération d'intellectuels, dite de la " nouvelle sensibilité », ou communautarienne-républicaine, réagira. Elle tentera de réhabiliter la singularité d'un passé québécois sans réhabiliter la Grande Noirceur. C'est un bilan à la fois théorique et politique des communautariens-républicains que nous voudrions effectuer ici : un bilan théorique, qui reprendra les principales thèses québécoises contemporaines sur la réhabilitation du passé, et un bilan politique, qui rappellera la difficile articulation entre la singularité québécoise (les valeurs) et son universalisme (la laïcité). Il n'est pas si facile de se débarrasser de la Grande Noirceur sans se débarrasser du passé québécois. 


\title{
Comment se débarrasser de la Grande Noirceur sans se débarrasser du passé québécois?
}

\author{
Jean-François Laniel \\ Faculté de théologie et \\ de sciences religieuses \\ Université Laval \\ Joseph Yvon Thériault \\ Département de sociologie \\ Université du Québec à Montréal
}

\section{Résumé}

La Grande Noirceur est fille de la Révolution tranquille. Pour affirmer la victoire de la modernité, les nouvelles élites québécoises noircirent exagérément le moment qu'elles venaient de quitter. Les historiens dits révisionnistes sonnèrent l'alarme : le passé québécois aurait été de tout temps "normal ». C'est à cette lecture qu'une nouvelle génération d'intellectuels, dite de la " nouvelle sensibilité ", ou communautarienne-républicaine, réagira. Elle tentera de réhabiliter la singularité d'un passé québécois sans réhabiliter la Grande Noirceur. C'est un bilan à la fois théorique et politique des communautariens-républicains que nous voudrions effectuer ici : un bilan théorique, qui reprendra les principales thèses québécoises contemporaines sur la réhabilitation du passé, et un bilan politique, qui rappellera la difficile articulation entre la singularité québécoise (les valeurs) et son universalisme (la laïcité). Il n'est pas si facile de se débarrasser de la Grande Noirceur sans se débarrasser du passé québécois. 
Le monde est vide depuis les Romains; mais leur mémoire le remplit et prophétise le nom de liberté. SAINT-Just, Discours à la Convention nationale, le 11 germinal an II (31 mars 1794)

\section{De la Grande Noirceur à la liquidation du passé québécois}

Ce sont les vainqueurs qui expliquent habituellement l'histoire, du moins dans un premier temps. Les vaincus sont déclassés, défaits, leur interprétation du monde jetée aux poubelles de l'histoire. "Le monde est vide depuis les Romains ", disait Saint-Just à la Convention nationale de 1794 pour expliquer comment l'ancien régime était déconsidéré, en nette rupture avec le neuf. Le récit qui surgit alors vise à expliquer les temps nouveaux comme une réinscription du progrès dans l'histoire. On reconnaîtra ici les grands récits des révolutions modernes, tant américaine que française. On pense, notamment, à History of the United States of America, from the Discovery of the American Continent de George Bancroft ou à L'histoire de France de Jules Michelet, auvres marquantes qui réinscrivent les révolutions respectives des États-Unis et de France dans l'histoire du progrès de l'humanité. Il faut un temps pour que surgissent des explications autres, pour que de nouvelles oppositions s'organisent, pour que les forces expulsées du changement n'apparaissent plus réactionnaires, mais, plus simplement, celles d'une tradition ayant façonné le présent.

C'est dans cette optique qu'il faut lire l'histoire de l'idée de la Grande Noirceur au Québec. Cette expression est apparue au moment où celui-ci était en marche vers la Révolution tranquille (attestée dès 1945 , reprise en 1966) ${ }^{1}$. Elle serait le récit confirmant la victoire de

${ }^{1}$ Alexandre Turgeon, Robert La Palme et les origines caricaturales de la Grande Noirceur duplessiste : conception et diffusion d'un mythistoire au Québec, des années 1940 à nos jours, thèse de doctorat (histoire), Université Laval, 2015. En une formule, chez 
la nouvelle technocratie ${ }^{2}$, la version inversée de la longue marche des technocrates ${ }^{3}$. Elle est, pour reprendre les termes de Jocelyn Létourneau, un vocable du récit populaire du Québec francophone, celui de son repli séculaire, celui quapprennent spontanément les jeunes Québécois à travers la mémoire familiale, celui qui est transmis dans la cinématographie historique (Séraphin, un homme et son péché), les téléséries (Les filles de Caleb), les romans (Le Survenant) ${ }^{4}$. Récit populaire qui explique beaucoup des attitudes politiques contemporaines, mais qui a aussi, comme nous le verrons, son pendant dans une littérature sociohistorique qui peut éclairer le parcours de l'idée de laïcité au Québec.

La Grande Noirceur désignait au départ une période restreinte, celle du gouvernement duplessiste, son conservatisme (1944-1959). Une période dite d'obscurantisme, réfractaire aux forces du progrès, qu'elles fussent culturelles, économiques ou politiques. Le sens de l'expression viendra très tôt à désigner toute la période du Canada français et de l'hégémonie du clergé (ultramontain, "clériconationaliste ", "intégriste ") sur la société (1840-1960). Alors que l'Occident se modernisait, le Québec aurait vécu une sorte de fixation dans le passé, en économie (agriculturalisme), en politique (antiétatisme), au cœur de sa mentalité (messianisme) $)^{5}$ " Un long hiver de la survivance ", dira même Fernand Dumont, pourtant empathique

Gilles Marcotte : "Il n'y avait pas grand-chose de grand dans cette Noirceur... " (Pierre Popovic, Entretiens avec Gilles Marcotte : de la littérature avant toute chose, Montréal, Éditions Liber, 1996, p. 58).

2 Jocelyn Létourneau, "La nouvelle figure identitaire du Québécois : essai sur la dimension symbolique d'un consensus social en voie d'émergence ", British Journal of Canadian Studies, vol. 6, n ${ }^{\circ} 1$ (1991), p. 17-38.

3 Jean-Jacques Simard, La longue marche des technocrates, Montréal, Les Éditions coopératives Albert Saint-Martin, 1979.

4 Film réalisé par Charles Binamé, 2002; série réalisée par Jean Beaudin, d'après le roman d'Arlette Cousture, 1990-1991; d'après le roman écrit par Germaine Guèvremont, 1945.

5 Michel Brunet, "Trois dominantes de la pensée canadienne-française : l'agriculturisme, l'anti-étatisme et le messianisme ", Écrits du Canada français, n ${ }^{\circ} 3$ (1957), p. 31-118. 
à l'existence historique du Canada français. On se souviendra du triptyque de Marcel Rioux dans La question du Québec (1966), l'un des premiers livres utilisant l'expression "Québec » pour désigner ce que l'on appelait auparavant le Canada français : « tradition, rattrapage, dépassement ». La Révolution tranquille était pour lui une "période de rattrapage ", le passage entre "la tradition " et son éventuel " dépassement " dans une sorte de Québec postmoderne avant la lettre. En 1960, le Québec sortait de la tradition, de la Grande Noirceur.

Lorsqu'elle est définie ainsi, la Grande Noirceur n'est pas dissociée du jugement que posent les analystes contemporains sur le parcours historique du Québec. Comprendre la Grande Noirceur, c'est nécessairement réinscrire celle-ci dans le récit du passé québécois effectué par des générations de sociologues ou d'historiens depuis la Révolution tranquille. Le débat sur la Grande Noirceur est symptomatique de la difficulté des intellectuels du Québec moderne à assumer le passé. C'est ce que ce texte vise à montrer. Il est utile d'observer comment le Québec a construit la mémoire de son passé afin d'en mesurer les conséquences contemporaines, jusque dans le débat sur la laïcité à la québécoise. De la Grande Noirceur à aujourd'hui, la religion catholique se trouvera sur notre chemin, celui que d'autres ont trouvé par elle bloqué, et qui ne cesse de se manifester.

La Grande Noirceur est une lecture accablante pour la mémoire du Québec français. En histoire, l'idée d'un Québec non pas uniquement traditionnel, mais d'un Québec historique impuissant s'est largement imposée dès le milieu des années 1950. Les successeurs de Lionel Groulx, par exemple Maurice Séguin, Guy Frégault, Marcel Brunet, voulurent rompre avec une histoire qui glorifiait la résilience du peuple canadien-français et de ses héros (plus grands que nature) face à un destin tragique. Les héros canadiens n'étaient ni héros ni saints, mais plutôt des "dépossédés et des outlaws ». Selon Groulx, la Conquête chez Frégault "n'aurait laissé [que] ruine dans son 
sillage $^{6} »$. De manière générale, ces nouveaux historiens pratiquaient une histoire et un nationalisme "négatifs ", voyant dans le passé québécois une suite ininterrompue de catastrophes et de défaites ayant décapité les forces vives de la nation ${ }^{7}$. Une historiographie de la terre brûlée, où le seul chemin possible est vers l'avant, sans retour ni repos possible. "Le plus important ", professait d'ailleurs Séguin, théoricien du néonationalisme, c'est d' "être distinct avant d'être différent : il faut exister séparément d'abord (avant d'avoir une personnalité collective) [...] la personnalité, l'originalité viennent nécessairement après le fait $d^{\prime}$ exister $^{8}{ }$.

Il est vrai que, pour les successeurs montréalais de Groulx (l'École historique de Montréal), tout cela s'inscrivait dans un projet de " reconquête ", une sorte d'appel au peuple pour qu'il se défasse de son immobilisme atavique. C'est ce que Marcel Rioux formulait par son appel au "dépassement ", un pari de l'arrachement, un dépassement de soi pour soi. Seulement, chez les nouveaux historiens, comme chez Rioux, à l'inverse de Groulx, il n'était plus possible de faire reposer cette proposition sur une histoire vivante du Canada français. Rioux récusera explicitement dans certains textes la "tradition " comme lieu possible de l'émancipation. Le passage, au milieu des années 1960, du vocable Canada français à Québécois est exemplaire à cet égard : le Canada français n'est pas le passé du Québec, du moins c'est un passé mort. Comme le dira É.-Martin Meunier, "il fallait oublier le Canada français, tout en ne cessant pas de critiquer para-

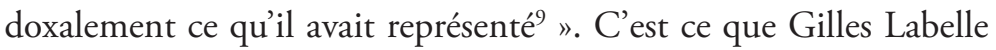

${ }^{6}$ Ronald Rudin, Faire de l'histoire au Québec, Québec, Éditions du Septentrion, 1998, p. 131-133.

7 Jean Lamarre, Le devenir de la nation québécoise selon Maurice Séguin, Guy Frégault et Michel Brunet (1944-1969), Québec, Éditions du Septentrion, 1993; Lionel Groulx, Dollard est-il un mythe?, Montréal, Éditions Fides, 1960.

${ }^{8}$ Maurice Séguin, Les normes de Maurice Séguin : le théoricien du néo-nationalisme, Montréal, Guérin, 1999, p. 161.

9 É.-Martin Meunier, « La Grande Noirceur canadienne-française dans l'historiographie et la mémoire québécoises : revisiter une interprétation convenue ", Vingtième Siècle : revue d'histoire, $\mathrm{n}^{\circ} 129$ (2016), p. 45. 
nommera la "fondation négative " du Québec moderne, mue par une " colère anti-théologique " aux multiples déclinaison $s^{10}$.

La critique de l'impuissance de la société canadienne-française fut encore plus virulente chez les historiens adversaires de l'École historique de Montréal : l'École de Laval (Marcel Trudel, Jean Hamelin et Fernand Ouellet) ${ }^{11}$. S'intéressant plus particulièrement aux retards socioéconomiques des Canadiens français (leur faible présence dans le monde de l'économie capitaliste, les inégalités de revenus, etc.), ils délaisseront l'explication (nationaliste) selon laquelle la Conquête et la domination anglaise seraient responsables de cet état de fait. Ces derniers concluront plutôt à la responsabilité de la mentalité canadienne-française, notamment celle de ses élites (essentiellement le clergé catholique). On trouvera, au même moment, chez le jeune Pierre Elliott Trudeau, une version plus politique de l'autoresponsabilisation canadienne-française dans la production de ce passé noir. C'est la mentalité canadienne-française qui aurait conduit le Canada français à une conception arriérée, ethnique de la démocratie, précisera-t-il, dans "Quelques obstacles à la démocratie au Québec ": "Ils refusèrent de se lier intérieurement par une "volonté générale" qui ne tenait pas compte du problème ethnique ${ }^{12}$ ".

Ce sont les sociologues qui illustreront le mieux l'ampleur de la condamnation du passé canadien-français ainsi contenue dans l'expression «Grande Noirceur ». On sait que la sociologie s'est

${ }^{10}$ Gilles Labelle, "Sens et destin de la colère antithéologique au Québec après la Révolution tranquille ", dans Jacques Palard, Alain-G. Gagnon et Bernard Gagnon (dir.), Diversité et identités au Québec et dans les régions d'Europe, Québec, Les Presses de l'Université Laval; Bruxelles, Peter Lang, 2006, p. 337-364.

11 Pour une mise à jour de la thèse des Écoles de Montréal et de Laval, FrançoisOlivier Dorais, "Classifier et organiser la production historiographique au Québec : réflexions critiques autour de la notion d'“école historique" ", Bulletin d'histoire politique, vol. 24, no 3 (2016), p. 158-176; "Marcel Trudel et Guy Frégault : regards sur une amitié intellectuelle ", Recherches sociographiques, vol. 57, $\mathrm{n}^{\text {os }}$ 2-3 (2016), p. 523-552.

12 Pierre Elliott Trudeau, "De quelques obstacles à la démocratie au Québec ", Le fédéralisme et la société canadienne-française, 1967, Montréal, Hurtubise $\mathrm{HMH}$, p. 111. 
largement construite sur l'opposition entre la tradition et la modernité (la sociologie est science de la modernité ${ }^{13}$ ). Selon cette lecture, les sociétés traditionnelles sont des sociétés communautaires, où les individus sont soudés mécaniquement, des communautés inscrites dans un univers de sens, inconscientes de leur historicité, renvoyant celle-ci à des forces extérieures à la société : Dieu, la tradition, la nature. Au contraire, les sociétés modernes sont des sociétés individualistes, où les individus sont connectés fonctionnellement, des communautés réflexives conscientes de leur historicité (elles affirment faire ou devoir faire leur propre histoire). Les premiers travaux sociologiques portant sur le Canada français seront fortement marqués par cette dichotomie. On pense, notamment, aux études des sociologues américains Horace Miner et Everett Hughes qui, dans le prolongement des travaux de l'école de Chicago, produiront des études marquantes sur le Canada français ${ }^{14}$. Ils verront le Canada français comme une société traditionnelle, une folk society ou encore une société ethnique, dans le meilleur des cas une communauté en transition vers le monde moderne. Dans la tradition américaine, la folk society était habituellement associée à un reste de traditionalisme présent dans une société résolument moderne, un atavisme lié à la provenance " paysanne " des immigrants, pour qui la religion joue un rôle prépondérant. Cette notion appliquée au Canada français ramenait l'entièreté de la société à la tradition. Le Canada français était une folk society, à l'image notamment des communautés autochtones du Yucatan mexicain étudiées par Robert Redfield.

13 Voir, à ce sujet, Robert A. Nisbet, La tradition sociologique, Paris, Presses universitaires de France, 1984.

14 Voir Léon Gérin, Le type agricole et social des Canadiens, milieux agricoles de traditions françaises, Montréal, Éditions Fides, 1948 ; Everett C. Hughes, French Canada in Transition, Chicago, The University of Chicago Press, 1943; et Horace Miner, "Le changement dans la culture rurale canadienne-française ", dans Marcel Rioux et Yves Martin (dir.), La société canadienne-française, Montréal, Hurtubise HMH, 1979, p. 77-99. Voir, à ce sujet, l'histoire que propose Gilles Bourque de la sociologie et de la société québécoises : "Société traditionnelle, société politique et sociologie québécoise ", Cahiers de recherche sociologique, n 20 (1993), p. 45-83. 
La riposte des sociologues québécois ne se fera pas attendre ${ }^{15}$. Elle s'effectuera par une double substitution dont la première sera de nature sémantique : le mot "Québec " se substituera à celui de "Canadien français » pour parler du groupe concerné. Cette transition se fait rapidement, au milieu des années 1960. Rioux nomme encore, en 1964, son objet d'étude Canada français; en 1969, il publie, La question du Québec. Politiquement, les États généraux du Canada français (1966-1969) affirmeront que le destin du Canada français passe dorénavant par l'État du Québec. Le Téléjournal de RadioCanada commencera alors à hiérarchiser le Québec dans la présentation de son bulletin de nouvelles : il y aura les nouvelles québécoises, les nouvelles canadiennes et les nouvelles internationales.

Lappellation de folk society ne tient plus car elle concernait la "société canadienne-française "; le Québec, c'est autre chose. On peut alors procéder à une autre substitution, plus théorique, celle de "société globale ». L'idée apparaît au même moment, notamment chez Rioux et Dumont ${ }^{16}$. Elle renvoie à l'existence d'une société moderne, mue par les grandes forces de la modernité (individualisme, capitalisme, technicisme) et traversée par des conflits de classes et d'identité (pluralité), une société pouvant aspirer à un haut niveau

15 En fait, le paradigme de la folk society est contesté dès les premiers travaux de Redfield, dans les années 1930. Il est accusé d'évolutionnisme et d'ethnocentrisme. L'apex de sa critique aux États-Unis surviendra au milieu des années 1950 et, à peine quelques années plus tard au Québec, notamment chez Philippe Garigue, mais également chez Marcel Rioux et Fernand Dumont. Si de nombreux sociologues québécois reprirent les grandes catégories et caractéristiques du paradigme moderniste popularisé par Redfield, Miner et Hughes, notamment par l'entremise de Jean-Charles Falardeau, il ne s'agissait néanmoins pas d'un pur calque; de nombreuses interprétations et appropriations eurent lieu, qu'il faudra étudier plus avant, car elles révèlent elles aussi les préoccupations et les espoirs d'un milieu, de sa tradition sociologique en gestation.

16 Gilles Bourque, Jules Duchastel et André Kuzminski, "Les grandeurs et les misères de la société globale au Québec ", Cahiers de recherche sociologique, nº 28 (1997), p. 7-17; Joseph Yvon Thériault, "La société globale est morte, vive la société globale ", Cahiers de recherche sociologique, nº 28 (1997), p. 19-35. 
d'historicité. Le Québec est une « société globale », le Canada français était une "folk society" ${ }^{17}$ ".

Mais, la folk society est-elle vraiment le passé du Québec, sa Grande Noirceur? D'une certaine façon, c'est ce que s'évertueront à nier les analystes (sociologues, comme historiens) dits révisionnistes du tournant des années $1980^{18}$. La dissimilitude des deux appellations (Canada français et Québec) facilitait en cela grandement la tâche. Ils continueront à associer le Canada français à une folk society, une société ethnique, mais relieront de moins en moins son caractère traditionnel au passé du Québec, le trait fondamental de cette société. Autrement dit, ils étendront l'idée de la société globale québécoise au passé québécois, une modernisation rétrospective, là où les révolutionnaires tranquilles proposaient une modernisation prospective, à conquérir plutôt qu'à reconnaître. Le gouvernement Duplessis, épicentre de la Grande Noirceur, aurait été un régime libéral (Bourque et Duchastel ${ }^{19}$ ). La ruralité québécoise (l'expérience tardive de la colonisation) et la famille québécoise auraient été des prototypes d'une modernité américaine (américanité) (Bouchard, Courville ${ }^{20}$. La pensée libérale de même que la modernité technique auraient pénétré le Québec au même rythme que la moderne Amérique (Lamonde, Roy ${ }^{21}$. Graduellement, on arrivera à l'idée que le Québec

17 Bien que, pendant quelques courtes années transitoires, ce fût la société globale canadienne-française que l'on étudia, notamment Fernand Dumont, "L'étude de la société globale canadienne-française ", Recherches sociographiques, vol. 3, $\mathrm{n}^{\text {os }} 1-2$ (1963), p. 277-294. Pour un exemple de transition ambivalente, Gérald Fortin, "Le Québec, une société globale à la recherche d'elle-même ", Recherches sociographiques, vol. 8, $\mathrm{n}^{\circ} 1$ (janvier-avril 1967), p. 7-13.

18 Voir Rudin, Faire de l'histoire au Québec.

19 Gilles Bourque et Jules Duchastel, avec la collaboration de Jacques Beauchemin et Pierre Plante, Restons traditionnels et progressifs, Montréal, Éditions du Boréal, 1988.

20 Serge Courville, Entre ville et campagne, Québec, Les Presses de l'Université Laval, 1990 ; Gérard Bouchard, Quelques arpents d'Amérique : population, économie, famille, Montréal, Éditions du Boréal, 1996.

21 Yvan Lamonde et Esther Trépanier (dir.), L'avènement de la modernité culturelle au Québec, Québec, Institut québécois de recherche sur la culture, 1986; Fernande Roy, Histoire des idéologies au Québec aux XIX et XX siècles, Montréal, Éditions du Boréal, 1993. 
n'a jamais été traditionnel. Comme l'affirmera le sociologue Gilles Gagné, le Québec serait né tout entier «moderne » sur le bateau de Champlain : « [L]e Québec fait partie de ces sociétés [...] dont toute l'histoire appartient à la dynamique de la modernité2 ". L'idée de la Grande Noirceur serait un leurre.

Exit la Grande Noirceur, mais, en même temps, exit la mémoire d'une histoire singulière. Car la lecture des écrivains modernistes révisionnistes aura tendance à banaliser le Québec (Rudin dira, à faire du Québec une "société normale "), à en faire, comme on le disait dans l'introduction au livre phare du révisionnisme, l'Histoire du Québec contemporain, un simple territoire où déambule par hasard un échantillon de l'humanité :

Le Québec que nous étudions ici est défini comme un territoire plutôt que d'après l'appartenance ethnique. Nous nous intéressons aux phénomènes qui sont survenus sur le territoire, aux hommes et aux femmes qui l'ont habité. Tout au long, le mot québécois est donc employé dans un sens très précis. Il désigne tous les résidents du Québec, que leur ancêtre soit venu du nord-ouest, il y a quelques milliers d'années, qu'il soit arrivé de France à l'époque de Jean Talon, qu'il soit un Écossais ayant traversé l'Atlantique en 1780, un Irlandais fuyant la Grande Famine, un juif tentant d'échapper aux persécutions de certains pays d'Europe de l'Est ou encore un Italien voulant sortir d'un Mezzogiorno qui a peu à lui offriri ${ }^{23}$.

Lévacuation du passé québécois se faisait ainsi au nom d'une froideur technique. Il fallait banaliser le passé québécois, le normaliser, le lessiver de son ethnicité, concevoir son parcours comme un parcours typique d'une américanité confondue avec la modernité. Bref, pour

22 Gilles Gagné, "Tradition et modernité au Québec : d'un quiproquo à l'autre ", dans Khaël Elbaz, Andrée Fortin et Guy Laforest ( dir.), Les frontières de l'identité: modernité et postmodernité au Québec, Québec, Les Presses de l'Université Laval, 1996, p. 68.

23 Paul-André Linteau René Durocher, Jean-Claude Robert et François Ricard, Histoire du Québec contemporain, t. 2 : le Québec depuis 1930, Montréal, Éditions du Boréal, 1989, p. 7, coll. « compact». 
en finir avec la Grande Noirceur, il fallait, comme le dira Gérard Bouchard, faire abstraction de la fausse conscience et des fausses représentations que la société se donnait d'elle-même. Il fallait étudier les soubassements infrastructurels de la société, non sa superstructure, " tout ce qui concerne l'exercice du pouvoir politique sur le plan paroissial a été exclu de notre enquête ", précisera-t-i ${ }^{24}$. Les travaux des penseurs " modernistes " en viendront à privilégier les grands processus de modernisation (la montée du capitalisme, l'industrialisation, la démocratie, la mondialisation) de préférence aux « idéologies » ou aux " représentations politiques », considérées comme événementielles. C'est ainsi que l'on peut comprendre le déclin de l'histoire politique ou nationale dans les départements universitaires au profit d'une histoire dite sociale, tout comme, par ailleurs, dans l'enseignement de l'histoire à l'école ${ }^{25}$. Nous aurions eu une Grande Noirceur, mais celle-ci n’aurait été qu’un épiphénomène.

L'insistance sur les processus de modernisation au détriment d'une histoire nationale, d'une histoire contextualisée, avec pour fil d'Ariane une intention, une tradition de débats, a ainsi eu pour effet de juger l'histoire à l'aune des processus universalisants, la singularité historique s'effaçant ainsi devant des procès de plus en plus abstraits. De façon générale, la modernité comme civilisation s’est déployée à partir de tels processus : la raison, les droits humains, la science. Même les procès plus sociologiques, le capitalisme, l'industrialisation, la mondialisation, n’ont pas, à proprement parler, de patries. Ils ne définissent pas la singularité d'une société. L'industrialisation ou encore la démocratie sont arrivées, à quelques années près, en même temps dans les sociétés occidentales. Autrement dit, ce sont des processus universalisants qui, lorsqu'ils deviennent les vecteurs de

${ }^{24}$ Bouchard, Quelques arpents d'Amérique, p. 482.

25 Éric Bédard, «L'histoire nationale au Québec : l'héritage impossible », dans Serge Cantin, Éric Bédard et Daniel Lefeuvre (dir.), L’histoire nationale en débat : regards croisés sur la France et le Québec, Paris, Riveneuve, 2010, p. 57-76, coll. "Actes académiques ». 
l'écriture de l'histoire, donnent de celle-ci une lecture abstraite. À un certain niveau d'abstraction, tous les chats sont gris.

C'est ainsi que l'objet Québec, sa Grande Noirceur, se voyait noyé dans la modernisation. Pour matérialiser l'analyse des processus d'abstraction modernes, l'analyse sociale devait renouer avec l'univers du sens, la superstructure qui rend compte du véhicule singulier par lequel se concrétisent les procès universalisants. La nation fut dans la modernité l'un des véhicules privilégiés permettant de rendre compte de l'histoire effective de ces processus. Du moins, c'est ainsi qu'interpréteront au tournant des années 2000 de nouveaux lecteurs du récit québécois que l'on associera à une "nouvelle sensibilité ", mais que nous aimons mieux nommer, pour des raisons qui deviendront évidentes, communautariens et républicains.

\section{Le retour imparfait du passé : communautariens et républicains}

En 1998, Ronald Rudin publie la version française de Faire de l'histoire au Québec (la version anglaise avait paru l'année précédente). La plupart des historiens du Québec des trente dernières années, affirme-t-il, ont exagérément normalisé le passé du Québec, jusqu’à rendre son histoire insipide. Ces historiens, qu'il appelle " révisionnistes ", de manière à confirmer la modernisation de leur société, auraient ignoré la singularité historique du Québec et décrié le travail des historiens nationalistes qui les avaient précédés. Rudin plaide pour le retour à une certaine histoire plus soucieuse des différences contextuelles et des subjectivités propres aux différents groupes qui forment la société.

L'année suivante, en 1999, la revue Société publie un numéro dont le thème est "Le chaînon manquant ». Deux jeunes sociologues, É.-Martin Meunier et Jean-Philippe Warren, y signent un long article, "L'horizon personnaliste de la Révolution tranquille », qui deviendra quelques années plus tard un livre au titre évocateur : Sortir de la "Grande Noirceur" : l'horizon "personnaliste" de la Révolution 
tranquille, préfacé par l'historien Éric Bédard ${ }^{26}$. Se pourrait-il, se demandent ces jeunes auteurs, que le Québec actuel soit plus l'héritier du Canada français que ne le donne à penser la lecture que nous en faisons depuis quelques années? Se pourrait-il que ce soit par le passé catholique (son horizon personnaliste) que nous puissions au mieux comprendre la modernité québécoise? "À trop faire équivaloir la Grande Noirceur à l'ensemble du passé canadien-français, aurait-on sacrifié le sens de la vie de nos ancêtres au seul profit d'un Grand Récit, celui de l'entrée du Québec dans la modernitée ${ }^{77}$ ? ", se demandera encore plus récemment Meunier. Il y aurait continuité (pas uniquement rupture) dans la trame historique qui part du Canada français et qui conduit au Québec de la Révolution tranquille.

Naissait ainsi ce que Stéphane Kelly pourra appeler, dès 2003, " une nouvelle sensibilité historique ». Cette nouvelle sensibilité implique un regard plus empathique sur le passé; le retour de thèmes négligés dans le passé récent, "l'histoire intellectuelle, l'histoire politique et l'histoire religieuse " et même la francophonie hors Québec; et la création de nouveaux lieux de publication : "Cahiers d'histoire politique au XX ${ }^{e}$ siècle, Bulletin d'histoire politique, Arguments, $M e n s^{28}$ ". C'est dans ce créneau que l'on pourra inscrire l'ouvrage de Jacques Beauchemin, L'histoire en trop (2002), qui constate «la mauvaise conscience " des intellectuels québécois contemporains et plaide pour une relecture de l'histoire québécoise qui tienne compte de la présence d'une «majorité historique ». Retenons aussi celui de

26 É.-Martin Meunier et Jean-Philippe Warren, Sortir de la "Grande Noirceur " : l'horizon "personnaliste » de la Révolution tranquille, préface d'Éric Bédard, Québec, Éditions du Septentrion, 2002.

27 É.-Martin Meunier, "Le casse-tête mémoriel de l'Église catholique au Québec ", dans Étienne Pouliot et Anne Fortin (dir.), Identité et mémoire des chrétiens : propositions au-delà du repli identitaire, Montréal, Éditions Fides, 2013, p. 33.

28 Stéphane Kelly (dir.), Les idées mènent le Québec, Québec, Les Presses de l'Université Laval, 2003, p. 1. Lire également Lucille Beaudry et Marc Chevrier, Une pensée libérale, critique ou conservatrice? Actualité de Hannah Arendt, d'Emmanuel Mounier et de George Grant pour le Québec d'aujourd'hui, Québec, Les Presses de l'Université Laval, 2007. 
Joseph Yvon Thériault, Critique de l'américanité (2002), qui voit dans la pensée contemporaine de l'américanité un amour immodéré pour la modernité et une réduction de l'histoire du Québec aux grands processus abstraits. La dénationalisation tranquille (2007) de Mathieu Bock-Côté décrit, tout en le déplorant, l'effacement du récit national dans l'historiographie québécoise. Mentionnons les deux ouvrages de Bédard, Les Réformistes (2009) et Recours aux sources (2011) où l'histoire, même celle de ceux qui se sont éloignés le plus du progressisme moderne, redevient un lieu d'enseignement et de réflexion pour la politique d'aujourd'hui. Il faudrait ajouter les travaux de Marc Chevrier, La République québécoise (2012), et de Danic Parenteau, Précis républicain à l'usage des Québécois (2014), qui retracent l'imaginaire historique républicain au Québec. Daniel Dagenais dans La fin de la famille moderne (2000), et Stéphane Kelly, dans À l'ombre du mur : trajectoires et destin de la génération $X$ (2001), décrivent les effets délétères de la modernité et de la technocratie (Révolution tranquille) sur la famille contemporaine et la génération X. Enfin, les écrits d'un Jocelyn Létourneau, notamment Le Québec entre son passé et ses passages (2010), même s'ils sont critiques à l'égard de l'histoire racontée du point de vue du récit national, en mettant en évidence le passé tortueux et singulier des Québécois et de leur imaginaire culturel, participent de cette " nouvelle sensibilité ». Tout comme, d'ailleurs, l'un des derniers ouvrages de Gérard Bouchard, Mythes sociaux et imaginaires collectifs (2014), dans lequel ce dernier, qui s'est longtemps identifié au paradigme moderniste et à l'histoire sociale, rappelle dorénavant l'importance des représentations collectives singulières dans l'histoire des sociétés neuves.

Au-delà d'une plus grande empathie pour le passé québécois, tous ces auteurs relèvent-ils d'un même paradigme? Serait-on face à une "sensibilité " commune? Christian Roy, dans la conclusion de l'ouvrage Les idées mènent le Québec, parle "d'uchronie »" sorte

29 Martin Petitclerc associe l'origine de l'expression « uchronie » chez les historiens québécois à Pierre Trépanier, qui lui aurait donné un sens essentiellement 
d'utopie inversée où l'on cherche dans le passé certains possibles, pour définir celle-ci. Jean-Philippe Warren parlera plutôt d'un tournant culturel des études (sociologiques) québécoises ${ }^{30}$. Mais, pour répondre à notre question initiale, cette empathie pour le passé renoue-t-elle avec la Grande Noirceur? Il faut immédiatement rejeter l'affirmation d'une réaction conservatrice ${ }^{31}$ parce que cette affirmation est habituellement une accusation, voire une insulte, plus qu'une analyse $^{32}$. Certes, comme le dit Meunier, il y a une position " intellectuelle virtuellement conservatrice " dans la nouvelle sensibilité33. Mais tout recours à l'histoire ou à la mémoire ne l'est-il pas? Cela ne conduit pas pour autant à un conservatisme politique, moral ou idéologique. L'uchronie peut servir à idéaliser le passé (conservatisme idéologique), à le critiquer (utopie moderniste) ou, encore, à y voir des possibles (une tradition vivante), ce qu' elle prétend habituellement faire. On pourrait décrire la présence de toute la gamme de ces attitudes chez les penseurs de la nouvelle sensibilité quoiqu'on y trouve difficilement l'idée du conservatisme comme retour au passé, une telle attitude étant insupportable aux modernes que nous sommes tous. Même dans l'ouvrage de Jean-Marc Piotte et de Jean-Pierre Couture, Les nouveaux visages du nationalisme conservateur au Québec, les "néo-conservateurs " répertoriés ${ }^{34}$ n'ont ni la même attitude face au passé québécois ni les mêmes positions

conservateur. Martin Petitclerc, "Notre maître le passé? Le projet critique de l'histoire sociale et l'émergence d'une nouvelle sensibilité historique ", Revue d'histoire de l'Amérique française, vol. 63, nº 1 (2009), p. 83-113.

30 Jean-Philippe Warren, «La sociologie du Québec francophone aurait-elle succombé (sans qu'elle le sache) aux Cultural Studies? ", Bulletin d'histoire politique, vol. 14, $\mathrm{n}^{\circ} 1$ (automne 2005), p. 237-247.

31 Petitclerc, " Notre maître le passé? ".

32 Jean-Marc Piotte et Jean-Pierre Couture, Les nouveaux visages du nationalisme conservateur au Québec, Montréal, Québec Amérique, 2012.

33 É.-Martin Meunier, "Une nouvelle sensibilité pour les "Enfants du concile" ?", dans Kelly (dir.), Les idées mènent le Québec, p. 105.

${ }^{34}$ Les auteurs répertoriés sont les suivants : Joseph Yvon Thériault, Jacques Beauchemin, Gilles Labelle, Marc Chevrier et Stéphane Kelly. 
politiques, et aucun n'adhère, à ce que nous sachions, à un conservatisme moral ${ }^{35}$.

On trouvera d'ailleurs dans les travaux associés à cette nouvelle sensibilité la réitération du dilemme qui est au cœur de ce texte et qui a été au cœur de la question du Québec depuis le début des années 1960 : comment se débarrasser de la Grande Noirceur sans se débarrasser du passé québécois? En effet, la nouvelle sensibilité n'a pas toujours tenté de réhabiliter le passé canadien-français ${ }^{36}$; elle a même été parfois son principal critique à partir d'un autre passé, celui de la République. C'est pourquoi nous définirons deux grandes écoles rattachées à la nouvelle sensibilité, deux écoles qui conduiront à des jugements différents sur la trame historique du Québec.

La première école est communautarienne, ou holiste, pour emprunter au vocabulaire sociologique. En sociologie québécoise, elle s'inscrit en continuité avec les travaux de Fernand Dumont dans lesquels on trouve un plaidoyer en faveur de la mémoire canadiennefrançaise. Genèse de la société québécoise est un livre, malgré son titre, qui parle exclusivement de la mémoire canadienne-française du Québec. En assumant la mémoire du Canada français, les communautariens reconnaissent une partie de la Grande Noirceur comme

35 Nous pensons ici à un conservatisme de type moral majority américain ou au catholicisme moral d'un Mrr Ouellet au Québec. En fait, seuls Jacques Beauchemin (Argument et Labrèque, 2016) et Éric Bédard (dès 2003 avec Xavier Gélinas), à notre connaissance, se sont adjoints le qualificatif « conservateur ", le premier, toutefois, après que celui-ci lui a été accolé avec insistance et en circonscrivant très précisément le sens qu'il lui donne, c'est-à-dire la défense d'un monde commun et une prudence politique, terrain sur lequel il rejoint Éric Bédard. Peut-être faudrait-il alors parler d'un conservatisme d'ici, à la François-Xavier Garneau, à la sensibilité arendtienne et burkienne, aux allures différentes de ce que l'on entend par cela en Europe et aux États-Unis, à l'instar du terme « nationaliste », d'ailleurs.

36 On lui a d'ailleurs reproché un certain apolitisme sociologique (Thériault, Critique de l'américanité) et une posture essentiellement critique et déconstructiviste (Beauchemin l'a accusée de "cracher dans la soupe de la Révolution tranquille "). L'objet " personnaliste » y est peut-être pour quelque chose : s'il permet de nuancer fortement le récit moderniste laïciste, en montrant ses origines catholiques, il participe néanmoins de la critique du Canada français traditionnel. 
une expérience historique effective du Québec. L'appel, chez un Jacques Beauchemin, à la mémoire et à la présence d'une "majorité historique " canadienne-française va dans ce sens, de même que le rappel d'une « intention nationale " comme « tradition vivante » dans les travaux de Joseph Yvon Thériault. Le premier ouvrage de Mathieu Bock-Côté, La dénationalisation tranquille, s'inscrit aussi dans cette trame. Il plaide pour le retour à une conscience politique et historique qui tiendrait compte de sa tradition nationale. En histoire, il faut bien y voir la trace d'un Lionel Groulx ${ }^{37}$. Ici, les historiens sont moins nombreux à recourir à l'expérience politico-communautaire du Canada français ${ }^{38}$. Le plus connu d'entre eux est Éric Bédard. Celuici voit toujours dans le passé canadien-français une source utile à la politique actuelle. En bref, l'appel à la mémoire renvoie à une trame historique qui est celle du Canada français, empreinte de la fragilité et de la petitesse de l'expérience historique québécoise.

Le deuxième recours à la mémoire est plus républicain. On est plus près ici du sens plein de l'uchronie : chercher dans le passé, même dans ses franges, un possible actualisable. Les analyses du passé républicain québécois de Marc Chevrier ${ }^{39}$ ou encore de Danic Parenteau ${ }^{40}$ sont caractéristiques de cette attitude. Ces travaux critiquent le nationalisme canadien-français traditionnel, qui n'aurait pas assumé son expérience républicaine, dont Chevrier trouve les fondements dans la Nouvelle-France coloniale. L'ouvrage de Stéphane Kelly, La petite loterie, va dans le même sens, comme l'ensemble de

37 Jean-François Laniel, «Petites sociétés, élites intellectuelles et "tradition vivante" : contribution à une sociologie des petites sociétés ", dans Mihaï Dinu Gheorghiu et Paul Arnault (dir.), Les sciences sociales et leurs publics : engagements et distanciations, Iași (Roumanie), Éditions Université Alexandru Ioan Cuza, 2013, p. 423-445.

${ }^{38}$ Mentionnons, entre autres, Michel Bock, Quand la nation débordait les frontières: les minorités françaises dans la pensée de Lionel Groulx, Montréal, Hurtubise HMH, 2004; Charles-Philippe Courtois, Trois mouvements intellectuels québécois et leurs relations françaises: l'Action française, "La Relève " et "La Nation " (1917-1939), thèse de doctorat (histoire), Université du Québec à Montréal, 2008.

${ }^{39}$ Chevrier, La République québécoise.

40 Parenteau, Précis républicain à l'usage des Québécois. 
ses travaux d'ailleurs. Selon lui, il y a un passé républicain caché dans l'histoire du Québec qu'il faudrait faire renaître. Gilles Labelle se plaint que la " colère anti-théologique ${ }^{41}$ " des Québécois, à la sortie de la Grande Noirceur, n'ait pas produit une réelle révolution républicaine, comme si le poids du courant théologico-catholique était encore trop puissant... ou trop peu; dans les deux cas, une fondation politique claire et positive fut impossible ${ }^{42}$.

Une telle perspective républicaine a non seulement comme effet d'éloigner l'analyse de la trame historico-communautaire (l'histoire effective) du Canada français, mais de rapprocher l'histoire du Québec des grands processus abstraits de la modernité, ce que nous avons plus haut associé à l'un des traits dominants du paradigme moderniste ${ }^{43}$. C'est moins l'histoire du Québec, ou encore le Québec comme véhicule de la république qui est nommé comme tradition, qu'une république abstraite, sans ancrage précis dans une histoire nationale. On pourrait dire qu'eux aussi en s'éloignant de la "Grande Noirceur ", s'éloignent de l'histoire effective du Québec. C'est en se référant à un tel républicanisme que l'on peut comprendre comment un Gérard Bouchard peut continuellement affirmer l'importance de la mémoire nationale dans l'interculturalisme, les valeurs auxquelles il fait référence étant essentiellement les propositions universalisables de la modernité,

41 Gilles Labelle, "La Révolution tranquille interprétée à la lumière du "problème théologico-politique" ", Recherches sociographiques, vol. 52, n 3 (2011), p. 849-880.

42 On trouvera une telle position, la critique de la société québécoise au nom d'une tradition institutionnelle plus inventée qu'effective, dans l'approche freitagienne d'un Éric Martin. Voir, notamment, "Le printemps contre l'hégémonie : la mobilisation étudiante de 2012 et le blocage institutionnel de la société ", Recherches sociographiques, vol. 54, $\mathrm{n}^{\circ} 3$ (2013), p. 419-450.

43 Nous passons outre ici puisque la question du rapport à la Révolution tranquille ou encore à la dimension générationnelle de la nouvelle sensibilité est moins centrale à notre propos. Il y a dans la nouvelle sensibilité une critique du caractère déshumanisant de la Révolution tranquille. Encore ici, la nouvelle sensibilité n'est pas monolithique, les communautariens sont moins critiques de la Révolution tranquille, y voyant une continuité de l'intention nationale (Beauchemin, Thériault), alors que les républicains y voient plutôt le signe d'une révolution humaniste bâclée (Kelly, Chevrier). 
c'est-à-dire celles de l'individualisme et de l'État abstrait ${ }^{44}$. Paradoxalement, Mathieu Bock-Côté, pourtant longtemps associé à un communautarisme conservateur, dans son dernier ouvrage intitulé Le multiculturalisme comme religion politique, emprunte cette direction. L'idéologie diversitaire serait une trahison des élites de gauche envers la grande tradition républicaine d'égalité citoyenne occidentale.

C'est ainsi que l'on peut comprendre les récents appels, comme on le précisera plus loin, au moment du débat sur la Charte des valeurs québécoises notamment, aux valeurs essentiellement modernistes du Québec (démocratie, égalité homme-femme, laïcité, respect de la différence). Ici, les belligérants s'entremêlent. Multiculturalistes comme lä̈cistes s'affrontent au nom des mêmes valeurs de la modernité, les uns pour dire qu'elles doivent conduire à un plus grand respect de la diversité (libéraux), les autres à une plus grande unité nationale (républicains), mais plus personne ne parle du véhicule (la communauté historique, l'histoire effective) par lequel de telles valeurs se sont historiquement concrétisées ${ }^{45}$. C'est ainsi également que l'on peut comprendre l'étrange alliance qui s'est produite au Québec au moment des débats sur la laïcité, entre une vieille laïcité républicaine héritière des modernistes de la Révolution tranquille, critique de la Grande Noirceur (le mouvement lä̈que) et les nationalistes dits républicains-communautariens, largement issus de la nouvelle sensibilité. C’est là aussi la mémoire québécoise, le passé québécois, qui a disparu dans ce débat. Voyons cela de plus près.

${ }^{44}$ C'est la critique que nous adressions au rapport de la commission BouchardTaylor. Voir Joseph Yvon Thériault, «Entre républicanisme et multiculturalisme : la Commission Bouchard-Taylor, une synthèse ratée ", dans Bernard Gagnon (dir.), La diversité québécoise en débat, Montréal, Québec Amérique, 2010, p. 143-156.

45 C'était le centre de ma critique (Joseph Yvon Thériault) des deux manifestes ( Manifeste pour un Québec pluraliste » et « Déclaration des Intellectuels pour la laïcité ") ayant circulé au Québec. Voir Joseph Yvon Thériault, "Le Devoir de philo - Identité : le manifeste de Burke ", Le Devoir, 10-11 avril 2010, [En ligne], [http://www.ledevoir.com/societe/le-devoir-de-philo/286700/le-devoir-de-philoidentite-le-manifeste-de-burke]. 


\section{La Charte : une synthèse ratée}

À l'évidence, le débat québécois sur la place de la religion dans l'espace public, amorcé avec la « crise des accommodements raisonnables » (2007-2008) et suivi par la Charte des valeurs québécoises (2013-2014), ne fut pas radicalement étranger au milieu québécois, à sa communauté politique et historique. La société en entier fut interpelée, les intellectuels furent appelés à se prononcer, les partis politiques furent sommés de prendre parti, tandis que les concepts, les valeurs et les trames narratives employés trouvèrent un (certain) écho dans le pays réel : une majorité de Québécois (51\%) et tout particulièrement de Québécois francophones (68 \%) étaient favorables à la Charte des valeurs québécoises ${ }^{46}$.

Pour autant, les termes du débat furent, pour l'essentiel, circonscrits dans la trame moderniste classique ou révisionniste, prospective ou rétrospective, même chez les promoteurs des "valeurs québécoises ". On pourrait dire qu'ils furent représentatifs d'une seule moitié de l'histoire effective de la laïcité au Québec. La référence à une trame historique où s'inscrirait clairement la tradition québécoise, tout particulièrement religieuse, fut des plus ténues. Pareille référence moderniste, principielle et universaliste ne fut à même d'offrir qu' une lecture limitée et partielle du rapport aussi bien historique que contemporain des Québécois à la religion, à la laïcité. En cela, les propositions laïques qui fusèrent lors du débat sur la Charte eurent de la difficulté à produire une synthèse intellectuelle et politique inscrivant les principes de laïcité, d'égalité homme-femme, de bien commun ou encore de liberté religieuse dans un cadre historique et culturel contextualisé, et ce, qu'il s'agisse des partisans du pluralisme libéral ou républicain (multiculturaliste ou interculturaliste), ou des partisans du républicanisme jacobin ou communautarien (strict ou nationaliste). Nous y reviendrons.

${ }^{46}$ Louise Leduc, "L'appui à la charte est maintenant majoritaire ", La Presse, 3 mars 2014, [En ligne], [http://www.lapresse.ca/actualites/dossiers/charte-de-lalaicite/201403/03/01-4744020-lappui-a-la-charte-est-maintenant-majoritaire.php]. 
Soulignons d'abord en quoi le débat sur la place de la religion dans l'espace public québécois ne saurait porter uniquement sur la définition et la hiérarchie de principes universels, bien qu'il s'agisse là d'une amorce de tri et donc de contextualisation. On lui saurait gré de porter également sur la tradition québécoise en matière de laïcité, telle qu'elle émerge concrètement de la présence continue et disputée du catholicisme majoritaire dans la société québécoise. C'est en effet en dialogue, harmonieux et conflictuel, avec une religion, le catholicisme, que s'est construite la conception culturellement partagée de la religion au Québec et de sa place en société. Autrement dit, on peine à comprendre le rapport effectif et voulu des Québécois à la religion sans donner à celle-ci un nom et une histoire qui lui donne sa texture ainsi que sa trace dans les lois et dans les mœurs.

L'historiographie et la sociographie modernistes présentées plus tôt nous y auront préparés, mais on ne manque pas, malgré tout, de s'étonner qu'une telle lecture sociohistorique de l'imprégnation de l'Église catholique dans la société québécoise, réfléchissant à la place et à la conception de la religion, soit largement absente des débats et des travaux sur la laïcité québécoise. Il faut dire que, jusqu’il y a peu, la mémoire de la Grande Noirceur était telle qu'il était même suspect de s'intéresser à l'objet religieux, et encore davantage au catholicisme : se déployait au Québec, expliquent Robert Mager et É.-Martin Meunier, « un processus de définition utopique d'un Québec nouveau à construire et la liquidation d'un passé religieux, qui s'est faite sans ménagement. Dans ce procès, ces sciences [de la religion] n'étaient pas neutres : souvent nées d'un arrachement aux perspectives et au contrôle d'une conception traditionnelle de la religion, elles incitaient leurs représentants à en déclarer la mort ou à en faire l'autopsie ${ }^{47}$ ". En tout état de cause, le débat sur la laïcité semble réitérer l'embarras

47 Robert Mager et É.-Martin Meunier, «L'intrigue de la production moderne du religieux au Québec », Globe : revue internationale d'études québécoises, vol. 11, $\mathrm{n}^{\mathrm{o}} 1$ (2008), p. 15-16. 
des Québécois à l'égard de leur passé, contre lequel n’est pas prémuni l'univers du savoir.

On s'étonnera alors moins qu'un débat sur la religion ait pour l'essentiel opposé juristes, philosophes et politologues - ou leurs outils et cadres d'analyse, que de la manne d'ouvrages sur la laïcité ou la neutralité religieuse de l'État, il se soit agi de combats de principes et d'interprétations essentiellement juridiques, qui ne furent qu'exceptionnellement mis en rapport avec la religion concrète (le catholicisme, l'islam), telle qu'elle existe, est vécue et est comprise au Québec ${ }^{48}$. Avant de faire référence à la religion, c'est d'abord à la seule « diversité culturelle ", " aux pratiques d'accommodement reliées aux différences culturelles " que firent référence les débats et les travaux savants ${ }^{49}$. Et il n'est pas certain que l'emploi plus récent de « religion » soit beaucoup plus que nominal ${ }^{50}$. La question est pourtant de taille, puisqu'aucune société n'entretient le même rapport à la religion ni ne l'aménage pareillement. Par exemple, les pays historiquement protestants, à travers la tradition juridique de la "secularity " et de la liberté de croire, présentent des similarités entre eux, tout en possédant de grandes variantes internes, à l'instar des pays de tradition catholique, où la «laïcité » et la liberté face au croire se sont davantage développées ${ }^{51}$.

48 Par exemple, Sébastien Lévesque (dir.), Penser la lä̈cité québécoise : fondements et défense d'une lä̈cité ouverte au Québec, Québec, Les Presses de l'Université Laval, 2014.

49 Par exemple, outre la commission du même nom, Gagnon (dir.), La diversité québécoise en débat. Il en est tout autrement des mémoires, issus de la société civile, déposés à la commission Bouchard-Taylor, qui, eux, firent essentiellement référence à la religion, sous de nombreuses formes. Voir, notamment, pour ce qui est du catholicisme, Louis-Charles Gagnon-Tessier, Catholicisme et postmodernité : analyses philosophiques à partir de débats publics québécois, thèse de doctorat (sciences des religions), Université de Montréal, 2014.

50 Par exemple, lorsqu'il s'agit d'opposer sur un même plan sociologique la religion de la majorité et la religion de la minorité.

51 Le terme "laïcité " n'existe d'ailleurs pas dans la littérature savante angloprotestante. Voir Jacques Zylbergerg, «Laïcité, connais pas : Allemagne, Canada, États-Unis, Royaume-Uni », Pouvoirs, nº 75 (1995), p. 37-52; Peter Berger, Grace Davie et Effie Fokas, Religious America, Secular Europe? A Theme and Variations, Aldershot, Ashgate Publishing, 2008. 
C'est précisément afin de rendre compte de ces différentes trames sociétales, de ces multiples variantes entre l'État et la religion en modernité, que l'usage de concepts tels " modernités religieuses multiples ", ou "multiples secularities " se révèle de plus en plus fréquent ${ }^{52}$. Ils invitent précisément à " historiciser " notre entendement de la religion, de la sécularisation, de la laïcité, toutes construites sur le plan sociohistorique ${ }^{53}$. Ils mettent en garde contre les paradigmes uniformisants, de nature téléologique, souvent inspirés des grandes nations modernes que sont la France et les États-Unis : la laïcité, la neutralité, la liberté religieuse. Autrement dit, avant d'être un débat de principes, le débat sur la laïcité porte (ou devrait porter), plus profondément, sur l'identité du Québec et l'actualité de sa mémoire religieuse. Voyons ce qu'il en est dans le débat sur la Charte.

Quatre grandes postures, quatre grandes coalitions et constellations, chacune susceptible, selon les auteurs, d'accents variables, notamment de croisements et d'alliances (ce qui nous semble le cas ici), sont apparues lors du débat sur la Charte des valeurs québécoises : le pluralisme républicain (1), le républicanisme communautarien (2), le communautarisme libéral (3) et le pluralisme libéral (4) (figure 1). Nous retrouvons, particulièrement, ces positions dans quatre manifestes, dont elles sont issues, de manière inductive plutôt que déductive, ce qui contribue à expliquer que les croisements sont

52 Danièle Hervieu-Léger, "Pour une sociologie des "modernités religieuses multiples" : une autre approche de la "religion invisible" des sociétés européennes ", Social Compass, vol. 50, no 3 (2003), p. 287-295; Marian Burchardt, Monika Wohlrab-Sahr et Matthias Middel (dir.), Multiple Secularities Beyond the West: Religion and Modernity in the Global Age, Boston, De Gruyer, 2015. Voir, plus généralement, les travaux de David Martin et de Françoise Champion.

53 Ce qui ne veut pas dire arbitraires ou que l'on peut déconstruire à loisir. Talal Asad, Formations of the Secular: Christianity, Islam, Modernity, Stanford, Stanford University Press, 2003; Philip S. Gorski, « Historicizing the Secularization Debate: An Agenda for Research ", dans Michele Dilon (dir.), Handbook of the Sociology of Religion, Cambridge, Cambridge University Press, 2003, p. 110-122; Philip S. Gorski et Ateş Altinordu, "After Secularization? ", Annual Review of Sociology, vol. 34 (2008), p. 55-85. 
favorisés, ainsi que des composés que la théorie ne manie pas d'emblée ${ }^{54}$.

\section{Figure 1}

\section{Constellations politico-intellectuelles relatives au débat québécois sur la laïcité}

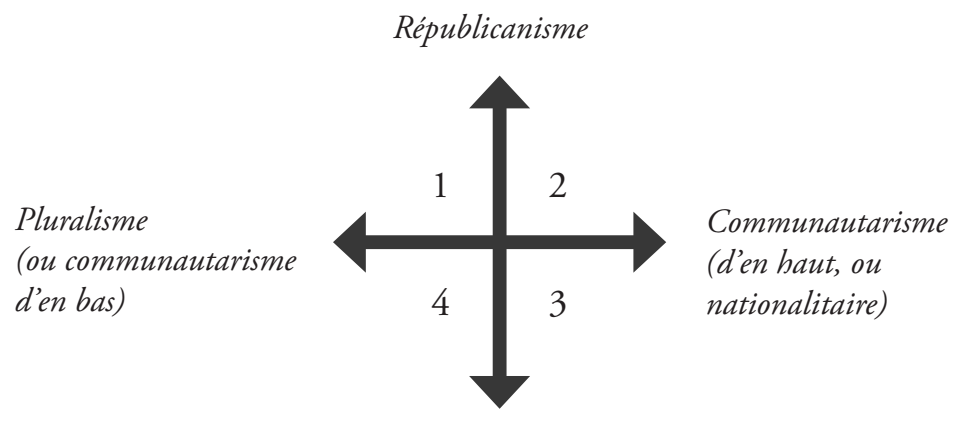

Libéralisme

Deux postures retiennent l'attention en raison de leur (sur)représentation politique et intellectuelle ainsi que de leur tentative, certes discrète, d'inscrire la laïcité québécoise dans un parcours québécois : (1) le "Manifeste pour un Québec pluraliste " (3 février 2010) et, (2) la "Déclaration des Intellectuels pour la laïcité - Pour un Québec laïque et pluraliste» $(16 \text { mars 2010 })^{55}$. On pourrait dire que l'un peut

${ }^{54}$ C'est dire que notre typologie se distingue de trois manières d'autres typologies (dont Lamy, 2016) : a) en prenant acte de la diversité interne du groupe " pluraliste ", à la fois républicain (Bouchard) et libéral (Taylor); b) en divisant le groupe républicain-communautarien ("républicain-conservateur » chez Lamy) afin d'inclure le point de vue catholique; c) en puisant d'abord aux manifestes plutôt qu'aux catégories de la philosophie politique : si les auteurs ont signé ces manifestes, c'est qu'ils ne se retrouvaient pas dans ceux qui étaient déjà lancés.

55 Notons, en effet, deux autres manifestes-types. Le texte de Joseph Yvon Thériault, "Identité : le manifeste de Burke ", publié dans la section Le Devoir de philo. (3) Il pourrait d'ailleurs récolter l'appui politique des mémoires présentés par 
revendiquer à son compte les recommandations du rapport BouchardTaylor, l'autre, le projet de loi 60 sur la Charte des valeurs québécoises. Ces deux textes, dans leurs efforts pour justifier la laïcité dans le cadre du débat politique, ont proposé une certaine lecture de l'histoire politique et religieuse québécoise. Tous deux modernistes (prospectif ou rétrospectif, classique ou révisionniste), ils ont réservé une place pour le moins timide au catholicisme, pouvant même aller jusqu’à l'annihiler. Déjà Michael Gauvreau écrivait au sujet de l'historiographie moderniste classique et révisionniste que, "dans ces deux récits le

l'Assemblée des évêques catholiques du Québec, déposés à l'occasion du projet de loi 60 et du projet de loi 62. Il affirme la nécessité de prendre en compte l'identité catholique du Québec, redoutant tout effet boomerang de la lä̈cité stricte ou ouverte sur les acquis institutionnels et symboliques catholiques et plaide pour le compromis politique ainsi que pour une véritable et concrète "politique d'intégration nationale ", au nom d'un "nationalisme positif". Nous le disons communautariste (d'en haut) en ce qu'il privilégie l'inscription dans une communauté nationale, historique et culturelle. Nous lui adjoignons le terme libéral en ce qu'il défend, comme Burke dont il se réclame, la liberté individuelle et celle de la société civile contre un État cherchant à régir d'en haut, abstraitement, la société (communautarisme (d'en haut) libéral). (4) Quant au "Manifeste pour un Québec inclusif ", dont les signataires incluent Daniel Weinstock, Jocelyn Maclure, Michel Seymour, Valérie Amiraux et David Koussens, on pourrait dire qu'ils ont à leur compte le projet de loi 62. Ils préferent la " neutralité religieuse " de l'État à toute forme de "laïcité ", car l'égale liberté religieuse individuelle prime, ce qui inclut le retrait des acquis institutionnels et symboliques catholiques, où toutes les religions individualisées devraient avoir droit de cité dans un espace public épuré de traces distinctives. La trame narrative est plutôt canadienne, d'où le peu d'attention que nous consacrons à ce manifeste et à sa vision de la laïcité. D'ailleurs, hormis la sortie de Charles Taylor, qui confirme la scission du groupe pluraliste (en républicains et libéraux), peu d'attention publique lui fut consacrée. Charles Taylor, « Le temps de la réconciliation ", La Presse, 14 février 2017, [En ligne], [http://plus.lapresse.ca/ screens/36c5c72e-28b9-49df-ba29-514fc56d647a\%7CpUtyV30bPPsb.html]. Nous la disons pluraliste (ou communautariste d'en bas) car l'égalité de toutes les religions devant la loi est sa priorité, assortie d'une critique vive de la majorité "privilégiée " par les institutions et le droit. Nous lui adjoignons le terme libéral car, à l'instar du multiculturalisme canadien, c'est en fait essentiellement à des individus que s'adresse ce pluralisme, dont (l'égale) liberté dans la société et au sein de l'État neutre est le projet principal (pluralisme (ou communautarisme d'en bas) libéral). 
catholicisme apparait [...] comme un figurant plus ou moins passif, jamais comme un acteur de premier plan dans l'histoire du Québec ${ }^{56}$ ". En ce sens, nous pourrions ajouter avec Jean-Jacques Simard que, dans ces deux récits, le Québec apparaît moins comme un " projet à l'horizon d'un peuple ", que le peuple québécois n'apparaît comme "un projet à l'horizon d'un État ${ }^{57}$ ", à construire plus ou moins abstraitement d'en haut.

Le premier récit, celui du "Manifeste pour un Québec pluraliste ", peut être dit pluraliste-républicain (1). On croit, en effet, y observer un républicanisme à l'américaine, lequel promeut le pluralisme intercommunautaire de la société civile et une identité nationale distincte et nouvelle, quoique perméable au substrat des interactions communautaires. Le second, celui de "Pour un Québec laïque et pluraliste ", peut être dit républicain-communautarien (2), bien qu'il nous apparaisse, nous le verrons, davantage comme un républicanisme à la française, qui institue une identité nationale nouvelle, distincte et imperméable aux particularismes communautaires, eux-mêmes renvoyés au privé. À l' "État faible » des ÉtatsUnis au nom des libertés individuelles et religieuses, la France privilégie, en effet, le modèle de l' "État fort ", au nom de l' "intérêt général $^{58}$ ». Tous deux n'en privilégient pas moins une identité nationale distinctement défendue par l'État, détachée d'une communauté culturelle particulière, à visée universaliste quoique diversement perméable à la société civile, qu'il s'agisse du «melting

56 Michael Gauvreau, Les origines catholiques de la Révolution tranquille, Montréal, Éditions Fides, 2008, p. 10.

57 En italique dans le texte. Jean-Jacques Simard, «La culture québécoise : question de nous ", Cahiers de recherche sociologique, no 14 (printemps 1990), p. 131; il cite Fernand Dumont, dans la première citation. Fernand Dumont, Le sort de la culture, Montréal, Éditions de l'Hexagone, 1987, p. 11.

58 Pierre Birnbaum, "Défense de l'État "fort" : réflexions sur la place du religieux en France et aux États-Unis ", Revue française de sociologie, vol. 52, n 3 (2011), p. 559-578. Voir, également, Bertrand Badie et Pierre Birnbaum, Sociologie de l'État, Paris, Éditions Grasset, [1979] 1982. 
pot " et du " wall of separation " américains ou de la France " une et indivisible ».

Le " Manifeste pour un Québec pluraliste » nous semble correspondre grosso modo à cette première vision continentaliste du Québec (1). À titre de "société neuve », le Québec participerait de la logique nord-américaine en matière de gestion de la diversité religieuse, conçue comme liberté de croire. À l'instar du pluralisme protestant américain, le Québec, par le biais de son inscription canadienne, aurait connu dès sa fondation un pluralisme religieux, protestant et catholique, reconnu par l'État, notamment dans le droit scolaire confessionnel constitutionnalisé en 1867, construisant de facto une laïcité ouverte dont il ne s'agirait plus aujourd'hui que de reconnaître de jure l'existence et, surtout, de l'étendre aux autres religions ${ }^{59}$. Peut-être faut-il y voir là la tentation annexionniste de la tradition politique des Rouges. La laïcisation du Québec se serait alors déroulée « silencieusement ${ }^{60}$ ", dans l' «impensé ${ }^{61}$ ", « implicitement ${ }^{62}$ ", « tranquillement " et " en douce ${ }^{63}$ ", par-delà les repré-

59 Gérard Bouchard, à la suite de Jack Jedwab, reconnaissait d'ailleurs que les principes interculturels n'ont pas été traduits en politiques au Québec (voir Jack Jedwab, "Le Québec est-il vraiment un État interculturel? ", Le Devoir, 25 février 2015, [En ligne], [http://www.ledevoir.com/politique/quebec/432789/le-quebec-estil-vraiment-un-etat-interculturel]; et Gérard Bouchard, "Précisions au sujet de l'interculturalisme québécois ", Le Devoir, 12 mars 2015, [En ligne], [http://www. ledevoir.com/societe/actualites-en-societe/434116/double-replique-precisions-ausujet-de-l-interculturalisme-quebecois].

${ }^{60}$ «En fait, tout se passe comme si les principes de séparation, d'égalité, de liberté de religion que tentent d'acquérir les grandes nations au prix de débats houleux, voire de combats, s'infiltraient silencieusement au Québec. Comme si le pragmatisme politique permettait de "faire" sans "dire" "(Micheline Milot, Lä̈cité dans le Nouveau Monde : le cas du Québec, préface de Jean Bauberot, Turnout, Brepols, 2002, p. 44).

61 Ibid., p. 19.

${ }^{62}$ Micheline Milot, "L'émergence de la notion de laïcité au Québec : résistances, polysémie et instrumentalisation ", dans Paul Eid et al. (dir.), Appartenances religieuses, appartenance citoyenne : un équilibre en tension, Québec, Les Presses de l'Université Laval, 2009, p. 67.

63 Louis Balthazar, "La laïcisation tranquille au Québec ", dans Jacques Lemire (dir.), La lä̈cité en Amérique du Nord, Bruxelles, Éditions de l'Université de Bruxelles, 1990, p. 31-42. 
sentations collectives d'une société régie par le monopole catholique, elles-mêmes jugées fausses ${ }^{64}$, car elles ne correspondaient ni au pluralisme religieux des territoires canadiens, québécois, voire américains, ni au droit qui encadre le catholicisme et le protestantisme. Ici, l'histoire de la laïcité québécoise se confond avec l'évolution " implicite » du droit canadien et québécois, d'une part, et la sublimation des groupes ethniques qui habitent le territoire, d'autre part. L'appel à une nouvelle " culture publique commune " autour de la langue française, défendue et instituée par une Charte, lui donne un hâle républicain, dit interculturaliste, se distinguant ainsi légèrement du Canada multiculturaliste et monarchiste, auquel contribue la laïcisation des figures coercitives de l'État et le retrait des symboles majoritaires des institutions publiques ${ }^{65}$. Le Québec est vu comme le cadre dans lequel évoluent différentes communautés culturelles et religieuses, dont les héritiers de la nation canadienne-française, chacune aspirant légitimement à reproduire ses différences. Leurs rencontres consubstantielles dans la langue française produiraient une nouvelle identité commune ${ }^{66}$. Le déisme du républicanisme

${ }^{64}$ Milot, Lä̈cité dans le Nouveau Monde, p. 20-21, 24. Toute une tradition juridique s'emploie d'ailleurs à opposer " laïcité réelle " à " laïcité discursive ", "laïcité juridique » et « laïcité du discours ».

65 Cette lecture de l'interculturalisme comme républicanisme discret ou, pourquoi pas, ouvert, étonne peut-être moins si elle est liée à l'américanité et au concept de société neuve, comme volonté innée, dite naturelle, de faire table rase, ce qui lui permet d'ailleurs d'adopter un point de vue pluraliste favorable au communautarisme d'en bas. Le Canada britannique et monarchiste apparaît alors bien davantage libéral-pluraliste. Nous croyons déjà observer ce républicanisme des sociétés neuves dans Thériault, Critique de l'américanité et " Entre républicanisme et multiculturalisme».

${ }^{66}$ L'interculturalisme traduirait une "volonté d'atténuer la dualité (ainsi que le rapport majorité-minorités) afin de réduire les frontières ou les clivages et de créer une culture commune [qui] doit composer avec le droit des membres de la majorité fondatrice et des minorités de rester attachés à leur héritage et à leur identité " (Gérard Bouchard, Linterculturalisme : un point de vue québécois, Montréal, Éditions du Boréal, 2012, p. 58). Toutefois, la neutralité des figures de coercition semble davantage un principe laïque qu'une concession à la majorité. C'est bien ainsi, en tout cas, qu'a interprété le tout Charles Taylor, pour expliquer son différend récent avec Gérard Bouchard sur cette question. 
américain est remplacé par la «francophonie » nord-américaine, tous deux, bien qu'à des degrés variables, jouant un rôle d'universalisation du pluralisme (religieux) de la société civile, ici reconnue et aménagée par la laïcité ouverte de l'État. On peut ainsi parler d'un modernisme révisionniste ou rétrospectif, puisque la Grande Noirceur comme la chrétienté canadienne-française n'existent plus que de manière épiphénoménale dans ce récit de l'américanité qu'elles ne structurent pas; il est tout en continentalité, en processus et en communautés empiriques, sans sujet ni médiation. Le Québec ayant de tout temps été pluraliste, la " laïcité discursive " de la population et de certains politiciens, arquée sur la mémoire de la Grande Noirceur et de la Révolution tranquille, serait davantage l'effet de " peurs " que d'une compréhension de la "laïcité réelle ». Exit le catholicisme comme dimension structurante, quand bien même négative, de l'imaginaire québécois et de son rapport (institué) à la religion. Un an avant la crise des accommodements raisonnables, Gérard Bouchard ne se demandait-il pas si, " en somme, pour caractériser cette dimension de l'histoire culturelle québécoise, faut-il dire : trop de religion? Ou trop peu ${ }^{67}$ ?"

Pour sa part, la « Déclaration des Intellectuels pour la laïcité Pour un Québec laïque et pluraliste " (2) prend acte de la mémoire et de l'expérience historiques du sujet québécois, mais en incluant la (seule) dimension négative du rapport au passé canadien-français. Elle correspond en cela à la perspective moderniste classique ou prospective, celle de l'opposition entre la Grande Noirceur et la Révolution tranquille, comme ambition de liquidation refondatrice de la tradition canadienne-française. L'évolution de la laïcité au Québec se comprend alors à partir d'une Église catholique monopolistique, s'imposant comme dépositaire de la nationalité canadienne-

${ }^{67}$ Gérard Bouchard, «L'imaginaire de la grande noirceur et de la révolution tranquille : fictions identitaires et jeux de mémoire au Québec ", Recherches sociographiques, vol. 46, n' 3 (2005), p. 430-431. On peut penser que le catholicisme a partie liée dans ce récit avec une " culture savante " détachée de la " culture populaire " (américaine). 
française et comme maître d'œuvre de ses institutions. Le passage du Canada français traditionnel au Québec moderne prend le visage de l'idéal-type français de la lutte entre l'Église catholique, le peuple et l'État québécois, c'est-à-dire d'un combat en faveur de la liberté face au croire. Il reproduit dès lors certains éléments de la "guerre des deux France ", opposant la France catholique à la France laïque, ici représentée par la tradition politique anticléricale des Rouges, du Mouvement laïque de langue française (MLF), puis du Mouvement laïque québécois (MLQ). En ce sens, le drame de la laïcité québécoise serait d'avoir cru cette lutte achevée au tournant de la Révolution tranquille : son ambition aurait en quelque sorte été dévoyée, à tout le moins retardée, jusqu'à aujourd'hui, telle est une des " méprises de la Révolution tranquille ${ }^{68} »$. Nulle surprise alors de retrouver cette formule historiographique sous la plume moderniste (classique) d'Yvan Lamonde, semblable en cela à celle (révisionniste) de Gérard Bouchard : " [L']équation $\mathrm{Q}=-(\mathrm{F})+(\mathrm{GB})+\mathrm{USA}^{2}$ - (R) est un moyen communicationnel de résumer une vision et une révision de l'identité du Québec ${ }^{69}$ ". Quoi qu’il en soit, ce républicanisme à la française n'attendrait plus que son " achèvement ", là où le républicanisme à l'américaine attendait sa « reconnaissance ». Et in fine, chaque fois, la variable religieuse, catholique, est écartée de la trame historique et politique.

En ce qu'il tablait sur un rapport à la religion inscrit dans la trame narrative québécoise, a contrario du pluralisme républicain (1), ce républicanisme à la française a pu favoriser, on le comprend, un rapprochement entre révolutionnaires tranquilles (Guy Rocher, Yvan Lamonde, Daniel Baril, etc.) et sympathisants de la nouvelle

${ }^{68}$ Voir Yvan Lamonde, L’heure de vérité : la laïcité québécoise à l'épreuve de l'histoire, Montréal, Del Busso Éditeur, 2010; Yvan Lamonde, "Malaise dans la culture québécoise : les méprises à propos de la Révolution tranquille », dans Guy Berthiaume et Claude Corbo (dir.), La Révolution tranquille en héritage, Montréal, Éditions du Boréal, 2011, p. 11-26.

69 Yvan Lamonde, «Penser l'américanité du Québec? ", Politique et sociétés, vol. 18, $\mathrm{n}^{\circ} 1$ (1999), p. 93. 
sensibilité historique (Jacques Beauchemin, Éric Bédard, Mathieu Bock-Côté et possiblement, en théorie, Marc Chevrier et Stéphane Kelly). Dès lors, républicaine-communautarienne (2), la Charte des valeurs québécoises proposait d'articuler les principes universalistes de la laïcité à la française avec un récit de la communauté historique québécoise, fondée d'abord contre la religion, selon le récit de la Révolution tranquille, mais aussi culturellement liée au catholicisme. La Charte des valeurs, au risque de nous répéter, obtint une majorité d'appuis dans la population québécoise et davantage encore parmi les francophones. "Derrière la laïcité, la nation ${ }^{70}$ ", pouvait ainsi écrire Mathieu Bock-Côté en 2008. Une synthèse républicainecommunautarienne semblait possible : «[L']idéal républicain tient donc dans ces tentatives concomitantes du respect des droits fondamentaux d'une part et, d'autre part, du maintien d'une identité nationale forte servant de foyer de convergence ${ }^{71}$ ", jugent Jacques Beauchemin et Louise Beaudoin. Les « implications concrètes " de cet idéal républicain, poursuivent-ils, « nous apparaissent avec plus de clarté. C'est bien, en effet, au nom de ce monde commun à construire que plusieurs appellent de leurs vœux une charte de la laïcité ${ }^{72}$ ". Un certain républicanisme à la française semblait ainsi permettre d'éviter de se débarrasser du passé québécois, d'éviter de pratiquer " la terre brûlée mémorielle ${ }^{73}$ ", ce que semblait également favoriser le maintien du crucifix à l'Assemblée nationale du Québec

${ }^{70}$ Mathieu Bock-Côté, «Derrière la laïcité, la nation : retour sur la controverse des accommodements raisonnables et sur la crise du multiculturalisme québécois ", Globe : revue internationale d'études québécoises, vol. 11, n 1 (2008), p. 95-114. Voir aussi Charles-Philippe Courtois, "La nation québécoise et la crise des accommodements raisonnables : bilan et prospectives ", Revue internationale d'études canadiennes, $\mathrm{n}^{\circ} 42$ (automne 2010), p. 283-306.

71 Jacques Beauchemin et Louise Beaudoin, "Le pluralisme comme incantation ", Le Devoir, 14 février 2010, p. C5.

72 Ibid.

73 Jacques Beauchemin, " 50 ans de révolution tranquille : quand les Québécois pratiquent la terre brûlée mémorielle ", Bulletin d'histoire politique, vol. 19, $n^{\circ} 3$ (2011). 
et l'ajout à la Charte d'une " clause patrimoniale " pour le catholicisme, tous deux ayant été " désémantisés » ou " neutralisés " par la sécularisation de la Révolution tranquille, donc compatibles avec la laïcité stricte.

Éric Bédard a tout particulièrement tenté d'articuler cet aspect de la synthèse républicaine-communautarienne, son inclusion ciblée de la variable catholique. "La sensibilité républicaine de la charte des valeurs québécoises est donc modulée par un conservatisme identitaire qui rechigne aux ruptures trop radicales avec le passé. Comme les autres sociétés occidentales, le Québec a été façonné par le christianisme. Même si les Québécois pratiquent peu ou plus du tout, plusieurs continuent de considérer la religion catholique comme un marqueur identitaire et ne souhaitent pas dépouiller nos édifices et nos places publiques des symboles de ce passé. " Avec la Charte, " le Québec tente une synthèse originale tout à fait en phase avec sa culture politique et son histoire ", puisant à même un ethos et un esprit républicain ayant animé certains patriotes et Rouges, sans pour autant recourir à la " laïcité revancharde " et " jacobine ${ }^{74}$ ".

Il nous semble toutefois que ce " compromis historique ${ }^{75}$ " fut des plus fragiles, autant dire une synthèse ratée. D’abord parce que la laïcité revancharde de certains baby-boomers fut malgré tout victorieuse, ensuite parce que la conception ainsi véhiculée du catholicisme patrimonial était pour le moins réductrice, enfin et plus fondamentalement parce que l'histoire ainsi récitée du Québec était profondément amputée car elle était tributaire d'une conception moderniste radicale de l'histoire.

Rappelons à cet égard quelques éléments contextuels du débat politique. La Charte trouva appui auprès de personnalités et de candidates péquistes jacobines très médiatisées telles Djemila Benhabib

${ }_{74}$ Éric Bédard, "Pour la charte des valeurs québécoises ", Argument, Exclusivités web, 2013.

75 Éric Bédard, "Pour un compromis historique ", Le Devoir, 12 septembre 2013, [En ligne], [http://www.ledevoir.com/politique/quebec/387208/pour-uncompromis-historique]. 
et Louise Mailloux, toutes deux favorables au retrait du crucifix de l'Assemblée nationale et toutes deux défaites aux élections de 2014. Mailloux considérait le baptême et la circoncision comme des "viols", sans que ses propos rapportés dans tous les médias ne fussent condamnés par Pauline Marois ${ }^{76}$. La Charte trouva également écho chez Lise Payette, qui se demanda, un soir d'élection, devant son écran de téléviseur où défilait la toponymie catholique du Québec, « combien d'années [il faudrait], avec un programme cohérent, pour changer les noms des villages et des petites villes du Québec ${ }^{77}$ ". Ajoutons que le PQ s'est converti tardivement au maintien du crucifix à l'Assemblée nationale, et qu'il aura fallu pour cela sa cuisante défaite électorale au profit de l'ADQ, en 2008, qui s'est présenté comme le défenseur de l'identité québécoise, en plein débat sur le sapin de Noël et le « joyeux Noël ». Et régulièrement, sous le gouvernement péquiste, a été exprimé le souhait de voir le crucifix retiré, tant par Bernard Drainville que par des ténors péquistes, dont Jacques Parizeau ${ }^{78}$. Peut-être, d'ailleurs, est-ce en raison de ce rapport de force en faveur des républicains laïcistes que l'avenir du patrimoine catholique a été si peu examiné et développé dans le projet de Charte. Et que le chef du Parti libéral du Québec, Philippe Couillard, quelques jours avant le début de la dernière campagne électorale, a pu dire aux électeurs des « régions » qu'il était le seul vrai défenseur du catholicisme ${ }^{79}$.

${ }^{76}$ Martin Ouellet, "Circoncision et viol : Pauline Marois refuse de condamner les propos de sa candidate », Le Devoir, 14 mars 2015, [En ligne], [http://www. ledevoir.com/politique/quebec/402677/circoncision-et-viol-marois-refuse-decondamner-les-propos-de-sa-candidate].

77 Lise Payette, «Quand la réalité frappe en plein front », Le Devoir, 8 novembre 2013, [En ligne], [http://www.ledevoir.com/politique/montreal/392088/quand-larealite-frappe-en-plein-front].

78 Geneviève Lavoie, "Le PQ favorable au retrait du crucifix ", Le Journal de Québec, 7 novembre 2013, [En ligne], [http://www.journaldequebec.com/2013/11/07/ le-pq-favorable-au-retrait-du-crucifix].

79 Il a récidivé en se prononçant en faveur du maintien du crucifix à l'hôpital SaintSacrement de Québec. 
Toujours est-il que l'avenir des lieux où sont imbriqués religion catholique et État québécois n'était pas précisé dans le projet de Charte, malgré la clause patrimoniale. Qu'allait-il advenir de ces centres pour personnes âgées avec leurs symboles, leurs messes et leurs aumôniers catholiques; de ces garderies subventionnées par l'État sises dans les églises catholiques, de ces facultés de théologie également financées par l'État ou, encore, de ces villages québécois où les églises sont utilisées aux fins du culte et pour des activités communautaires séculières, partagées par l'Église et la municipalité? Se profilait ici l'effet boomerang que nous redoutions (Joseph Yvon Thériault) dans le "Manifeste de Burke ", où la laïcisation stricte aurait pour plus grand effet le retrait de toutes les présences publiques du catholicisme (3). Sans parler de l'opposition que la Charte aurait immanquablement reçue des tribunaux canadiens, le Parti québécois allant même jusqu'à refuser de divulguer ses avis juridiques, mais aussi de s'engager à employer la clause dérogatoire. De même, le statut du catholicisme ainsi défendu n'était pas sans susciter le malaise de l'une des premières concernées, l'Église catholique, qui mit en garde contre la «folklorisation " de son patrimoine ( $\mathrm{M}^{\text {gr }}$ Fournier), à ses yeux bien plus que culturel, mais religieux. Elle préféra même voir le crucifix retiré du Salon bleu qu' "instrumentalisé $e^{80}$ " au service d'un républicanisme fort éloigné de la prudence des petites nations ${ }^{81}$.

C'est toutefois en histoire (religieuse) que la synthèse républicaine-communautarienne (2) révèle davantage sa fragilité. En effet, le récit moderniste dans lequel s'inscrit de manière privilégiée le projet de Charte des valeurs québécoises obscurcit non seulement le passé religieux canadien-français, mais néglige ses prolongements dans le Québec post-révolutionnaire tranquille. Ce récit n’intègre la

80 «Les évêques ne s’opposeraient pas au retrait du crucifix de l'Assemblée nationale ", Ici Radio-Canada, 19 septembre 2013, [En ligne], [http://ici.radio-canada.ca/ nouvelle/632894/eveques-charte-valeurs-quebecoises].

81 À ce chapitre de la prudence, qu'est-ce qui laisse croire que la " colère antithéologique " puisse se transsubstantier en transcendance républicaine, toute positive? 
dimension catholique que comme repoussoir ou faire-valoir, jamais en elle-même, du lieu de son historicité. En ce sens, nous y reviendrons, la Charte a peut-être pour mérite d'avoir montré les limites (et l'aboutissement du processus) de la "religion culturelle ", sous forme caricaturale ${ }^{82}$.

L'histoire du Canada français ne peut, en effet, être lue dans la perspective minoritaire des quelques patriotes républicains (il faut bien exclure ici les réformistes romantiques) et surtout des Rouges (anticléricaux et annexionnistes, rapidement décimés) qu’à condition de cesser d'être une histoire populaire, une histoire du sujet historique québécois, et de n'être plus qu'une histoire des procès universalistes de civilisation. L'histoire du Canada français est incompréhensible sans placer en son centre l'affinité élective entre l'ultramontanisme et le nationalisme canadien-français, entre un ultramontanisme aussi " institutionnel » que " populaire ${ }^{83}$ ", fruit d'un inédit renouveau religieux collectif, militant en faveur de la liberté de religion au fédéral afin de mieux servir son autonomie nationale et sociétale au provincial, auprès de son " peuple élu ${ }^{84}$ ». Car, au risque de rappeler l'évidence, et n'en déplaise aux analogies totalitaires, ce catholicisme populaire évoluait dans un cadre démocratique libéral; il ne tenait que tant et aussi longtemps que le peuple électeur le voulait bien, ce dont étaient conscients les laïcistes du MLF et ce qui les enjoignait à une patiente persévérance ${ }^{85}$. Ce catholicisme national et sociétal se prolongea

${ }^{82}$ Cette thèse est plus longuement développée par É.-Martin Meunier, «L'ancrage du catholicisme au Québec et sa déliaison progressive : une sociologie historique de l'exculturation ", dans Solange Lefebvre, Céline Béraud et É.-Martin Meunier (dir.), Catholicisme et cultures : regards croisés Québec-France, Québec, Les Presses de l'Université Laval, 2015, p. 21-44.

83 Guy Laperrière, "Vingt ans de recherche sur l'ultramontanisme ", Recherches sociographiques, vol. 27, $\mathrm{n}^{\circ} 1$ (1986). Voir aussi les travaux de René Hardy, Roberto Perin, Louis Rousseau, Nive Voisine et Christine Hudon.

${ }^{84}$ Jean-François Laniel, "L'Église-nation canadienne-française au siècle des nationalités : regard croisé sur l'ultramontanisme et le nationalisme ", Études d'histoire religieuse, vol. $81, \mathrm{n}^{\text {os }} 1-2$ (2015), p. 15-37.

85 Collectif, L'école lä̈que, Montréal, Éditions du Jour, 1961. 
jusqu'au cœur du Québec contemporain, sous des atours progressistes, cette fois ${ }^{86}$. Et la décléricalisation effectuée par la Révolution tranquille n'a pas eu pour corollaire la déconfessionnalisation du Québec, encore moins sa sécularisation complète. L'Église catholique demeura bien présente dans le système public confessionnel québécois jusqu'au tournant des années 2000, et l'enseignement catholique y fut offert jusqu'en 2008. Ces huit années ont d'ailleurs servi, selon le mot du ministre de l'Éducation de l'époque, François Legault, à éviter « une rupture trop radicale " avec la «tradition confessionnelle » de l'école

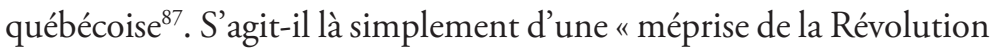
tranquille "?

Rappelons qu'encore en 1998-1999, 81,3 \% des élèves du primaire et $55,9 \%$ des élèves du secondaire suivaient le cours d'enseignement moral et religieux catholique ${ }^{88}$, et ce, certes au nom de la transmission d'une identité culturelle, mais aussi d'un bagage éthique, de "valeurs ${ }^{89}$ ". Plutôt que de pratiquer la laïcité ouverte ou stricte, c'est la " confessionnalité ouverte " que pratiqua l'école québécoise après la Révolution tranquille, suivant une " pédagogie de la conviction libre ", au nom " du développement intégral de la personne ». S’il en résulta un catholicisme « culturel » tendanciellement

${ }^{86}$ Martin Roy, Une réforme dans la fidélité : la revue Maintenant (1962-1974) et la "mise à jour " du catholicisme québécois, Québec, Les Presses de l'Université Laval, 2012.

${ }^{87}$ Micheline Milot, «École et religion au Québec : une laïcité en tension ", Spirale : revue de recherches en éducation, $\mathrm{n}^{\circ} 39$ (2007), p. 168.

${ }^{88}$ Jacques Palard, «Religion et politique au Québec : entre distance et mémoire ", dans Claude Sorbets et Jean-Pierre Augustin (dir.), Valeurs de sociétés : préférences politiques et références culturelles au Canada, Québec, Les Presses de l'Université Laval, 2001, p. 22-48.

89 Micheline Milot, Une religion à transmettre? Le choix des parents : essai d'analyse culturelle, Québec, Les Presses de l'Université Laval, 1991. On s'étonne d'ailleurs de l'interprétation donnée à ces entretiens et surtout de sa postérité dans le rapport Proulx. La dimension culturelle et éthique de l'enseignement religieux désiré a servi, en partie, à justifier l'abandon des cours d'enseignement religieux, au profit des cours d'éthique et de culture religieuse, mais c'était déjà cet enseignement qui était prodigué, dans une matrice catholique, et c'est de lui que parlaient les parents, qui continuaient à envoyer leurs enfants au cours d'enseignement religieux et moral... 
" culturalisé ", une sorte de religion civile où chacun inscrit librement sa subjectivité, même profane ${ }^{90}$, ce catholicisme culturel ne se résume pas pour autant à un slogan, à un drapeau... à un patrimoine. Il est également une éthique de la justice, de la tolérance et de devoirs civiques ${ }^{91}$. En cela, la crise des accommodements raisonnables survient au moment même où le catholicisme sort définitivement des écoles publiques québécoises, dans un contexte plus large d'exculturation ${ }^{92}$, là où il contribuait à " intégrer la personnalité collective québécoise ${ }^{93}$ " et comme persistent à le faire les rituels de passage et l'identification confessionnelle largement majoritaires ${ }^{94}$. Dans pareil contexte de " sécularisation accélérée ${ }^{95}$ ", faut-il s'étonner des réactions populaires et politiques aux débats récents sur la religion? Citons Louis Rousseau, qui fait écho au rapport " amour-haine ${ }^{96}$ » des Québécois à l'égard

${ }^{90}$ Jean-François Laniel, "Qu'en est-il de la "religion culturelle” ? Sécularisation, nation et imprégnation culturelle du christianisme ", dans Lefebvre, Béraud et Meunier (dir.), Catholicisme et cultures, p. 143-168; Jean-François Laniel, "La laïcité québécoise est-elle achevée? Essai sur une petite nation, entre société neuve et république ", dans É.-Martin Meunier (dir.), Le Québec et ses mutations culturelles : six enjeux pour le devenir d'une société, Ottawa, Les Presses de l'Université d'Ottawa, 2016, p. 423-474.

91 Voir, par exemple, sur l'enseignement catholique Stéphanie Tremblay, « Religions et éducation scolaire au Québec : une cartographie des enjeux politiques et sociologiques ", Diversité urbaine, vol. 9, n 1 (2009), p. 59-71.

92 É.-Martin Meunier, "L’ancrage du catholicisme au Québec et sa déliaison progressive : une sociologie historique de l'exculturation », dans Lefebvre, Béraud et Meunier (dir.), Catholicisme et cultures, p. 21-44.

93 Raymond Lemieux, "Le catholicisme québécois : une question de culture ", Sociologie et sociétés, vol. 22, nº 2 (1990).

${ }^{4}$ Il existe d'ailleurs plusieurs similarités entre les cours d'enseignement moral et religieux et d'éthique et de culture religieuse, à ceci près, ce qui est loin d'être négligeable, qu'est aboli chez le second le lieu d'où s'énonce l'éthique, plus englobant que quelques principes relatifs au respect de la religion d'autrui.

95 "Accélérée ", c'est-à-dire volontairement rapide, à mesure que s'accentuent les appels à une nouvelle "culture publique commune ", auxquels contribuent les pressions de la Charte canadienne et les ressacs du syndrome postréférendaire. Nous reprenons l'expression de Milot, "École et religion au Québec ».

96 É.-Martin Meunier et Jean-François Laniel, « Congrès eucharistique international 2008 : nation et catholicisme culturel au Québec : signification d'une recomposition religio-politique ", Sciences religieuses $=$ Studies in Religion, vol. 41, n 4 (2012), p. 595-617; É.-Martin Meunier, Jean-François Laniel et Jean-Christophe Demers, 
de la religion qua catholicisme : dans ces débats sur la religion, se fait jour « la crainte de la perte d'une référence identitaire essentielle, et son opposé, la crainte du retour sur la scène publique d'une institution de régulation et de contrôle qui confisque les libertés acquises ${ }^{97}$ \%. Disons de façon plus positive que, comme souci d'une certaine identité catholique québécoise et de ses valeurs " progressistes " à la québécoise, car il ne s'agit pas d'abord d'une réaction, mais plutôt d'une intention froissée, on peut comprendre aussi bien l'intérêt plus prononcé des Québécois pour la Charte des valeurs québécoises que l'insuffisance de celle-ci à emporter leur pleine adhésion dans un véritable élan d'enthousiasme.

\section{Comment se débarrasser de la Grande Noirceur sans se débarrasser du passé québécois?}

Avant toute chose, en cherchant à lire autrement qu'avec les seules lunettes modernistes (classiques ou révisionnistes, prospectives ou rétrospectives) le passé lointain et contemporain du Québec, en intégrant pleinement l'autre moitié de son histoire, notamment celle du catholicisme d'ici. Du Canada français au Québec moderne, de la doctrine ultramontaine à l'éthique personnaliste, de l'" Égliseinstitution " à l' "Église-peuple de Dieu" ", de la droite à la gauche, la tradition vivante québécoise allia l'utopie d'une société catholique à un projet de société francophone. Les traces laissées par cette

"Permanence et recomposition de la "religion culturelle" : aperçu socio-historique du catholicisme québécois (1970-2006) ", dans Robert Mager et Serge Cantin (dir.), Modernité et religion au Québec : où en sommes-nous?, Québec, Les Presses de l'Université Laval, 2010, p. 79-128.

${ }^{97}$ Et le religiologue de poursuivre : "J'appartiens au courant nationaliste engagé dans la recomposition d'un Québec issu de l'histoire longue et en faveur de l'inclusivité citoyenne. Ce qui demeure "impensé" dans ce courant, c'est la place de la religion. Or, ce "non-lieu" de l'identité historique est théoriquement et pratiquement insoutenable pour une majorité qui se reconnaît dans un projet de continuité » (Louis Rousseau, "La laïcité québécoise en régime de religiosité culturelle", Vivre ensemble, vol. 19, n 65 (2012), p. 3-4).

98 É.-Martin Meunier, Le pari personnaliste : modernité et catholicisme au XXe siècle, Montréal, Éditions Fides, 2007. 
tradition vivante permettent de suivre le fil d'une intention collective soluble ni dans le républicanisme antireligieux à la française ni dans le républicanisme pluraliste à l'américaine, encore moins dans le pluralisme libéral à la canadienne. Elle fait sourdre une référence autrement plus riche, complexe, enracinée et vivante que le précipité routinier des vainqueurs de la Révolution tranquille ${ }^{99}$, et sans laquelle demeure incompris le comportement politique des Québécois en matière de religion.

Il s'agirait d'inscrire dans une politique la trace d'une tradition nationale qui se serait historiquement institutionnalisée, par sédimentation (l'articulation d'un projet national et d'un pluralisme scolaire, par exemple). Il s'agirait aussi de poursuivre la trame institutionnelle par laquelle une société a historiquement répondu à la question de la diversité et de tracer le chemin par lequel elle peut continuer à y répondre, de donner plus d'importance à la tradition politique, à la communauté politique comme le lieu, ou comme l'horizon, où les citoyens peuvent débattre de l'universalisme des modernes et de leur tradition nationale. Nous ne saurions dire de manière plus précise, toutefois, ce à quoi ressemblerait une politique qui se développerait en s'inscrivant, non en fonction d'une opposition pluralisterépublicain / républicain-communautarien, mais dans l'histoire effective d'une tradition nationale vivante. Depuis la Révolution tranquille, on l'a vu, le passé québécois est couvert d'un voile, celui de la Grande Noirceur.

En cela, il n'est pas interdit de penser qu'à défaut d'une politique clé en main ${ }^{100}$, une sensibilité historique intégrant le passé québécois, et notamment sa dimension catholique dans sa dialectique avec le politique et la modernité, soit néanmoins de nature à inspirer un nationalisme positif et ainsi une véritable politique nationale d'intégration, en ce qu'elle permettra de comprendre le rapport effectif

99 Joseph Yvon Thériault, "Politique et démocratie au Québec : de l'émergence de la nation à la routinisation du souverainisme ", Recherches sociographiques, vol. 52, $\mathrm{n}^{\circ} 1$ (2011), p. 13-25.

100 Il ne saurait être question d'un déterminisme théologico-politique schmidtien. 
des Québécois à la religion, ce qui n'est pas de peu d'importance en plein débat démocratique sur la place de celle-ci en sociétée1 ${ }^{101}$ Un projet à l'horizon d'un peuple, cette fois, plutôt qu'un peuple à l'horizon d'un État, quand bien même évoquerait-il la grandeur américaine, française et, plus récemment, canadienne.

Après tout, qui aurait cru, l'heure des bilans législatifs venue, que la seule synthèse en acte du débat sur la laïcité serait la motion votée à l'unanimité par laquelle « l'Assemblée nationale réitère sa volonté de promouvoir la langue, l'histoire, la culture et les valeurs de la nation québécoise, favorise l'intégration de chacun à notre nation dans un esprit d'ouverture et de réciprocité et témoigne de son attachement à notre patrimoine religieux et historique représenté par le crucifix de notre Salon bleu et nos armoiries ornant nos institutions » (2008) ? Qui l'aurait cru il y a à peine quelques années, au moment du rapport Proulx et de la déconfessionnalisation des écoles québécoises? Et il y a une soixantaine d'années, au moment du rapport Parent et de la décléricalisation de la société québécoise? À moins que la dialectique de la terre brûlée et de la Terre promise, de la modernisation étatique et de la création d'un peuple nouveau, ne se soit depuis enrayée ${ }^{102}$. À moins que la sécularisation n'ait ici trouvé son cran d'arrêt ${ }^{103}$.

101 On peut se réjouir que, dans la plus récente mise à jour de leur bibliographie sélective sur le catholicisme québécois, les historiens Christine Hudon et Guy Laperrière aient ajouté 180 nouveaux titres pour la seule période allant de 2010 à 2015. Notons aussi que près de la moitié des 494 titres que compte la bibliographie ont été publiés entre 2006 et 2015. Deux nouveaux onglets thématiques ont même été ajoutés pour tenir compte des nouveaux champs d'intérêt : "laïcité " et " catholicisme culturel".

102 Encore et toujours, malgré l'absence de souveraineté et en vue d'elle, il s'agit de créer un nouveau peuple québécois, toujours plus moderne, c'est-à-dire toujours moins distinctement reconnaissable. L'américanité comme tabula rasa souverainiste, disait Yvan Lamonde. Sur ce processus, Marie McAndrew, « Projet national, immigration et intégration dans un Québec souverain : dix ans plus tard, l'analyse proposée tient-elle toujours la route? ", Sociologie et sociétés, vol. 38, n 1 (printemps 2006), p. 213-233. Après «feue l'unanimité »(G. Pelletier, 1960), "feue la québécitude" (É.-M. Meunier, 2016)?

103 C'est une hypothèse en faveur de laquelle les débats européens sur la place des symboles chrétiens dans les institutions publiques, notamment dans la désormais célèbre affaire Lautsi, offrent des arguments. 
En fait, jusqu’à présent, le débat sur la laïcité québécoise a eu tendance à réactualiser la question de la Grande Noirceur plutôt qu’à l'effacer, à se débarrasser du passé québécois plutôt qu’à l'assumer. L'opposition entre une position républicaine et communautarienne s'est largement estompée. Ou plutôt, de peur du communautarisme d'en bas, le communautarisme s'est de plus en plus défini par rapport au républicanisme, ce qui a accentué son caractère universaliste laïque, individualiste et étatiste, par opposition à un communautarisme ancré dans une tradition nationale. Que ce soit dans sa dimension nationale ou religieuse, c'est la difficulté de poser une tradition d'ici, une tradition vivante, que dévoile le recours contemporain à la Grande Noirceur. 